\title{
Labyrinthe
}

23 | 2006 (1)

Quatre chercheurs de l'étranger

\section{La mise en scène du droit}

Le corps inconnu dans la décision démocratique

\section{Ludger Schwarte}

\section{CpenEdition}

Journals

Édition électronique

URL : http://journals.openedition.org/labyrinthe/1156

DOI : $10.4000 /$ labyrinthe. 1156

ISSN : 1950-6031

Éditeur

Hermann

\section{Édition imprimée}

Date de publication : 15 janvier 2006

Pagination : 31-40

ISBN : 2-9526131-0-9

Référence électronique

Ludger Schwarte, «La mise en scène du droit », Labyrinthe [En ligne], 23 | 2006 (1), mis en ligne le 23 juillet 2008, consulté le 19 avril 2019. URL : http://journals.openedition.org/labyrinthe/1156 ; DOI : $10.4000 /$ labyrinthe. 1156 


\section{LA MISE EN SCÈNE DU DROIT* Le corps inconnu dans la décision démocratique}

Ludger SCHWARTE

\section{3 - Portée et possibilité : acteurs et orateurs dans la procédure légale ${ }^{1}$}

Les communautés de droit sont formées sur les différents niveaux [de légitimation ${ }^{2}$. Dans certaines situations, il peut être plus vraisemblable que l'on parvienne à conclure un contrat de mariage avec un canari qu'avec un Homo sapiens mal disposé. À l'époque moderne, on a réussi à faire de «l'homme», en tant qu'individu, le point de départ du droit; mais les représentations du droit et de ses capacités peuvent s'exprimer de manière cohérente tout en considérant les esclaves comme de simples quadrupèdes ${ }^{3}$. De fait, il n'existe aucune loi de la nature qui nous pousse à accepter un quelconque patrimoine génétique ou qui détermine notre sélection naturelle.

Tandis que la formation des communautés de droit peut être conduite à partir d'une réflexion sur la situation de base idéale pour la conclusion des contrats (voir par exemple les critères de Rawls), la situation de la cour de justice est bien différente. On doit pouvoir y représenter quelque chose qui n'est pas visible au moment de la conclusion du contrat.

\footnotetext{
* Extrait de «Die Inszenierung von Recht : der unbekannte Körper in der demokratischen Entscheidung», p. 119-125, dans Ludger Schwarte, Christoph Wulf (éd.), Anthropologische Dimensionen der Rechtsphilosophie, Munich, Wilhelm Fink, 2003, p. 93-139.

1. Nous suivons la numérotation de l'édition allemande.

2. Dans les paragraphes précédents, Ludger Schwarte distingue trois niveaux de légitimation du droit auquel correspondent trois interrogations différentes : un niveau systématique (le système juridique estil cohérent?), un niveau politique (le droit sert-il les intérêts de la communauté et de l'individu?) et, enfin, un niveau que nous qualifierons d' « esthétique » qui concerne la « construction théâtrale » du droit (la procédure a-t-elle été bien menée?). Le sens de ce paragraphe 3 est de mettre en évidence la nécessité actuelle de dépasser l'approche purement systématique et politique de la justice. La première est injustifiable et mène au relativisme, la seconde ne tient pas compte du droit tel qu'il se fait dans les tribunaux [Ndt.].

3. Il en est ainsi de la Lex Aquila. « On a fait abstraction de ce que les uns sont des hommes et les autres des bêtes. Seule importait la propriété. », Uwe Wesel, Juristische Weltkunde. Eine Einführung in das Recht. Francfort-sur-le-Main, Suhrkamp, 1984, p. 52.
} 


\section{1 - Porte-parole, participant et inactif}

Dans les procédures actuelles, il existe une différence caractéristique entre les orateurs et les participants (les serviteurs, le public, dans une certaine mesure les prévenus ainsi que les victimes). Les participants sont défendus par des porte-parole, bien qu'ils aient aussi la possibilité de s'exprimer. Ils sont la plupart du temps parfaitement capables d'agir, mais la prétendue rationalité du procès leur impose le silence. L'étendue de leurs actes se borne à se laisser défendre et à observer. Mais il y a d'autres actions outre l'acte de représentation - que peuvent (ou sont censés pouvoir) accomplir lors du procès certains participants, auxquels on ne peut normalement attribuer une faculté d'agir: les embryons, les animaux, les morts, les personnes sous tutelle. Ils se manifestent, interviennent par exemple comme intention ou matériel de preuve, et se tiennent dans un rapport à des lois qu'ils ne connaissent pas.

Ici, la fonction de représentation est étroitement liée à l'exigence de rendre le droit objectivable et permanent, et à celle de mobiliser la force de la loi, là où la personne concernée (rhétorique ou physique) manque d'une véritable puissance de réalisation. Il est néanmoins nécessaire que quelqu'un porte pour elle cette force. Et il me semble qu'il n'y a pas de contradiction entre le droit objectif et la rhétorique. Le problème n'est pas, selon moi, que dans les cours d'assises le droit, la rhétorique et la politique s'engrènent; en effet, ces catégories-là imprègnent aussi toute autre forme de décision contractuelle entre professionnels du droit.

La question est de savoir si et comment on est défendu. Et celle-ci doit être soumise à l'examen du caractère public de ce processus. L'articulation de la relation entre la représentation, le caractère public et la capacité d'action n'est fixée que par le jugement; mais elle doit être effective pour que celui-ci, lors duquel le propre et l'impropre s'entrechoquent, bascule. Ainsi, lors du processus d'invention du verdict, il est nécessaire que les circonstances de l'incapacité d'agir chez certains participants paraissent contingentes. Dans la plupart des cas, cela est le point de départ de la procédure (par exemple lors d'un meurtre). Juridiquement, il importe seulement que les orateurs débattent de ces circonstances. (Ce qui organise le déroulement du procès, ce sont les intérêts des survivants, éventuellement ceux de la communauté, et non pas la vérité du meurtre). Et comme, selon moi, il n'est pas souhaitable d'être 


\section{La mise en scène du droit}

représenté par celui qui a la prétention de le faire, il est plus sensé d'exiger un mandat direct.

\section{2 - Représentation, liturgie et rituel}

Si le droit lui-même doit rendre justice, alors il est nécessaire qu'il le fasse sous serment. Un droit quasi divin exige par conséquent une liturgie du procès. Le respect de la forme est plus important que l'issue du conflit, raison pour laquelle une fête serait souvent plus efficace qu'une longue procédure. Les conflits sont ici résolus quand s'affirme la validité universelle de la loi par le respect de la forme de l'office juridique, c'est-à-dire aussitôt que les parties adverses assument le rôle qui leur est prescrit. Pour que la validité universelle de la loi s'impose au travers de cette liturgie, il n'est pas nécessaire de savoir si elles articulent un quelconque intérêt à la solution du conflit.

Les procédures de la représentation, de la liturgie du procès et de l'identification ne sont pas les seules à déterminer qui entre en ligne de compte comme possible participant, c'est-à-dire en tant que sujet potentiel du droit (il n'y a pas que le droit qui décide de manière immanente). Les techniques scientifiques de production du visible et des normes intègrent dans la définition de ces dernières celles qui conviennent et aussi celles qui, en cas de doute, doivent être préservées, tout comme les substantivations politiques et religieuses des valeurs. Les critères juridiques de l'humanité, de ce qui est condamnable et de la souffrance (etc.) mettent en évidence le fait que la procédure juridique produit son propre «nous ». Il est possible que ce «nous » ait une origine proprement rituelle.

Ce qui est essentiel dans la procédure et les conduites juridiques ne me semble pas être la construction ou la réaffirmation d'un « nous » juridique - à la différence des techniques de cohésion et d'identification des autres systèmes sociaux (le législatif, l'exécutif) -, mais la clôture de réalités, le «transfert de soi» en une zone extérieure à ces réalités, la dissolution de ce «nous » dans des actes définis ${ }^{4}$.

\footnotetext{
4. Dans Les Guêpes d'Aristophane, Philocléon ne peut s'empêcher de juger et érige à son domicile un tribunal devant lequel il accuse son chien d'avoir volé du fromage; à la fin, il met un terme à ses accusations et se met à danser. Seule la danse peut se substituer au jugement car elle marque, elle aussi, un temps mort au sein des lois du présent.
} 


\section{3 - Protection des choses et incapacité à commettre des fautes}

Le «nous » sur lequel s'appuie la procédure pénale suppose que tous les participants à la procédure peuvent agir et fauter. Quelqu'un est accusé à raison, ou quelqu'un se plaint à tort. Il s'ensuit que seul celui qui, par sa propre résolution uniquement, en affecte un autre ${ }^{5}$, doit en être empêché par des sanctions pénales. L' «être sans voix et sans défense », la chose juridique, ne devient pas un sujet libre lorsqu'il est soustrait à l'intervention et aux intérêts cognitifs d'autrui - comme l'a proclamé Habermas lors d'une récente intervention -, mais quand il peut se prononcer pour ou contre tel ou tel autre traitement ${ }^{6}$.

\section{4 - La signification du procès pour la décision}

Le procès, compris comme la reconstruction d'un cas, a été décomposé par Hegel selon deux niveaux: l'établissement des preuves et le jugement. La reconstruction juridique du déroulement des faits que l'on juge ne sert pas seulement à réunir, comme dans un laboratoire, les éléments importants d'un processus. Elle sert aussi à esquisser et comprendre les alternatives (sans quoi il n'y aurait ni cas, ni même jugement) $)^{7}$.

\footnotetext{
5. Selon le principe de John Stuart Mill, « le seul aspect de la conduite d'un individu qui soit du ressort de la société est celui qui concerne autrui. Quant à l'aspect qui le concerne simplement lui-même, son indépendance est, en droit, absolue. L'individu est souverain sur lui-même, son propre corps et son propre esprit», De la liberté [trad. Fabrice Pataut], Paris, Presses Pocket, 1990, p. 40.

6. Pic de La Mirandole écrivait que l'homme était «arbitrarius plastes et fictor ». De nos jours, Jürgen Habermas incarne l'objection de la sculpture contre son créateur. Au nom de la liberté à l'autodétermination - et pour le compte des embryons faibles et sans voix -, il lutte contre les manipulations génétiques. Cependant, c'est d'abord cette biotechnologie qui fait de l'homme son propre statuaire - et l'émancipe par là de l'évolution et du hasard de la reproduction. Il n'est pas seulement « fixé » et «fait » par la technique génétique. En tant qu'être aux origines ancestrales, c'est seulement quand il entre dans la réciprocité sociale qu'il se situe en dehors de la fixation et du lien sanguin.

7. Sur la question de la rationalité des codes de procédures valides, Habermas se montre hésitant. C'est assez pour lui que des principes semblables à ceux du jury aient une chance dans l'établissement des faits et l'administration de preuves, lorsque celle-ci «est mise en scène comme interaction entre les personnes présentes » (259). Mais en même temps, il lui suffit à l'évidence que l'appréciation juridique de cet état de fait puisse être fondée et présentée ouvertement par le tribunal, même si, par là, il reconnaît aussi parfaitement que «la production du jugement continue d'être l'apanage du pouvoir professionnel des juges seuls ». (Droit et démocratie. Entre faits et normes, Paris, Gallimard, 1997, p. 259-260, trad. Rainer Rochlitz et Christian Bouchindhomme.) Curieusement, l'argument de Hegel selon lequel
} 


\section{La mise en scène du droit}

Dans la principale œuvre de Habermas consacrée à la philosophie du droit, Droit et Démocratie, un mécanisme n'est légitimé comme noyau procédural du principe d'une intégrité juridiquement garantie que s'il est essentiel à la détermination des rapports entre « les corps et le droit ». Il écrit:

[...] en tant que mécanisme destiné à élargir les relations concrètes de reconnaissance et à les transformer en une relation de la reconnaissance réciproque qui soit abstraite et de forme juridique, on se sert de la forme réflexive de l'activité communicationnelle, et plus particulièrement de la pratique argumentative qui demande à tout participant qu'il adopte les perspectives de tous les autres participants ${ }^{8}$.

De manière assez évidente, le droit surgit ici de comportements langagiers sur lesquels se fondent les relations concrètes de reconnaissance. Mais la possibilité de ces dernières ne repose pas seulement sur la rationalité du langage, mais aussi sur l'adoption d'autres perspectives qui existent déjà concrètement sur le plan corporel. La réunion des corps - ici considérés comme les membranes d'une reconnaissance réciproque au sein d'un même auditoire, forme par conséquent la rationalité sensible à partir de laquelle doit être fondée la procédure d'invention du droit. De la réunion de ces surfaces sensibles résultent d'abord tous les consentements qu'il est possible de formuler par le biais du langage, et de là, l' accord de tous les citoyens avec la constitution - à condition bien sûr qu'on ne présuppose pas déjà positivement le « droit comme un tout ».

\section{1 - Droit et théâtre : en quoi le tribunal diffère-t-il d'une scène de théâtre?}

Les codes de procédure ne règlent pas le processus sélectif d'engendrement et d'élimination d'autres possibilités, ni l'élaboration propre-

\footnotetext{
aucune personne formée au droit n'est qualifiée pour l'administration des preuves et que, par conséquent, le jugement devrait être l'expression de la « liberté subjective » du citoyen envers le droit, ne reçoit aucune attention de la part de Habermas. Stephen Todd a légitimement souligné qu'à Athènes les membres des dikastères [subdivision du tribunal de l'hélié composée de 600 membres tirés au hasard, Ndt] étaient plus que de simples jurés, car ils ne s'exprimaient pas seulement vis-à-vis d'un fait, mais aussi vis-à-vis du droit. Au fond, cela vaut pour toute sentence légale : celle-ci est toujours une appréciation du droit et pas seulement une estimation du cas.
}

8. Habermas, op. cit., p. 245. 


\section{Labyrinthe, $n^{\circ} 23$}

ment dite de la décision, mais la présentation de l'élaboration de la décision. La théorie du droit procédural pur ne revendique pas la conscience intersubjective de la vérité, mais seulement la valeur d'une procédure. Comme au théâtre, le contenu persuasif ne réside pas dans le fond de la décision, mais dans le fait consenti que l'on doit décider de cette manière là seulement.

Niklas Luhmann en conclut:

Voilà probablement la théorie cachée de la procédure: il serait possible en plongeant la personnalité dans un jeu de rôle de s'emparer d'elle, de la transformer et de la motiver à accepter des décisions ${ }^{9}$.

La mise en place de l'individuel à travers les masques de caractère ou l'air officiel, à travers la sémantisation du corporel, les séries d'actions et les rapports réciproques des acteurs entre eux, au regard de la totalité de la procédure, a pour but de capter la bienveillance non seulement des participants, mais aussi de l'auditoire.

Bien évidemment, cette esthétique de l'effet ne vaut qu'à l'intérieur d'un certain schéma de représentation, et ceci aussi bien pour un code de procédure bien défini que pour un modèle dramatique précis. Si l'on généralisait, on pourrait introduire l'idée que la pièce de théâtre entend représenter des possibilités, et la justice des vérités. Avec un autre concept de théâtre, à savoir la théâtralité, on pourrait parfaitement concevoir le procès comme un genre dramatique, alors même que certaines pièces sont principalement conçues comme des condamnations de certaines vérités. Un retour sur le tribunal populaire athénien nous permet aussi d'exhiber - outre des motifs purement formels et institutionnels - une différence entre justice et spectacle: la justice installe celui qui juge dans la réalité de ce qui lui est présenté, et expose ce processus à un plus large public, tandis que la pièce inclut le public, et expose ce processus à ceux qui jugent.

À ces deux types d'actions correspondent deux concepts de public. Dans un cas, le jugement est pratique, dans l'autre, il est esthétique. D'un côté, on produit la discontinuité d'une réalité, tandis que, de l'autre, cette réalité est généralisée et universalisée. Les corps et la mémoire sont

9. Dans La Légitimation par la procédure, Presses de l'université Laval-Éditions du Cerf, Laval-Paris, 2001, p. 80, trad. Lukas K. Sosoe et Stéphane Bouchard [modifiée par nos soins, Ndt]. 


\section{La mise en scène du droit}

produits au théâtre ${ }^{10}$. Le tribunal, lui, brise la mémoire, efface les blessures. Et vu que toutes les discontinuités sont dangereuses, les tribunaux sont le terme d'un «nous » et ne peuvent nullement servir à la constitution d'un corps social, mais ils sont à éviter du point de vue de la sécurité juridique.

\section{2 - Pourquoi juger?}

Se soustraire à la procédure était déjà dans l'Athènes antique un objet de la législation: il y avait des pénalités pour les procès tramés de manière injustifiée ${ }^{11}$.

Considéré du point de vue de la décision, chaque procès est une simple préhistoire.

Le procès canalise les processus de décidabilité, et doit pour cela comporter tant d'éléments d'indécision (matériels, chaotiques, contingents, quotidiens) qu'il s'apparente à l'extrajudiciaire comme étant sa répétition. Dans cette répétition s'insinuent des éléments qui perpétuent l'extrajudiciaire, de telle sorte que le conflit puisse être poursuivi indéfiniment au sein du tribunal et seulement dans les conditions fixées par ce dernier. Cela est une démarche de «désincarnation», ou plutôt de «fictionnalisation».

La décision, le jugement, est comme la saisie, la signature d'un contrat, le marquage ou la mise à distance stratégique, un revers extérieur, la production d'une mesurabilité, le terme de ce qui va de soi.

\section{3 - Ouverture et liaison}

Au moment même où le jugement tisse un lien entre une règle et un événement qui se trouve simultanément produit dans son unité, se crée une ouverture: la souveraineté du jugement marque dans le même temps

10. François Lyotard critique la théâtralité freudienne (Économie libidinale, Paris, Éditions de Minuit, 1974). Sa revendication d' « une théâtralité sans référence » sonne comme un contre-projet nietzschéen à une naissance de la morale à partir de la douleur de la mémoire. Voir Lyotard, Ibid., p. 29, 32 et 34. 11. Celui qui n'arrivait pas à recueillir un cinquième des voix des jurés devait s'acquitter d'une pénalité financière conséquente, lorsqu'il avait intenté un procès au nom de la communauté. Voir Tuttu Tarkiainen, Die Athenische Demokratie, Artemis, Zurich, 1966. p. 248. 


\section{Labyrinthe, $n^{\circ} 23$}

l'innocence et l'illégalité de la situation «d'avant». En effet, le jugement est toujours une modification de la règle et une divulgation de l'anarchie qui est immanente à son concept d'action.

La performance que constitue l'instauration du droit est confirmée par les frémissements et les craintes du public. Ainsi, Jacques Derrida écrit:

L'origine de l'autorité, la fondation ou le fondement [...] de la loi sont ellesmêmes une violence sans fondement. [...] Même si le succès de performatifs fondateurs d'un droit [...] supposent des conditions préalables et des conventions préalables (par exemple dans l'espace national ou international), la même limite «mystique» ressurgira à l'origine supposée desdites conditions, règles ou conventions - et leur interprétation dominante ${ }^{12}$.

À la différence de la législation, l'aporie de la jurisprudence réside, à travers l'application du droit, à accorder de temps à autre de la légitimité à ce qui est illégal. La projection scénique de la suspension du jugement dans la procédure - procédure qui doit consciencieusement accumuler du pouvoir en vue du monopole de la décision -, reproduit, afin de pouvoir véritablement juger, cette «origine de la règle».

\section{5 - La cour : une zone de non droit?}

La cour siège ensuite précisément lorsque la légalité de l'action apparaît comme n'étant plus évidente. Il y a deux types d'actions qui confirment cette légalité: celles qui se montrent conformes à la règle-comme le respect des règles de circulation routière - ou bien celles qui se tiennent en dehors de la zone du licite et de l'interdit. Respirer ou digérer, par exemple. Bizarrement, quand on mange, copule, dort ou chante, on pousse déjà dans une zone d'interdiction ce qui repose à peine à la base du concept d'action (l'action, c'est ce qu'on jugule?). Le jugement met nécessairement en évidence l'injustice de la loi, car il doit supposer que la loi n'avait pas une validité univoque en ce qui concerne la conduite à tenir, lorsque deux sujets moraux s'affrontent à propos d'une règle (qu'ils se sont eux-mêmes donnée), jusqu'à ce que le jugement assiste la loi et accrédite le droit.

12. Dans Force de loi. Le fondement mystique de l'autorité. Paris, Galilée, coll. « La philosophie en effet », 1994, p. 34. «La guerre est la violence légitime par excellence», ibid., p. 95 sqq. 


\section{La mise en scène du droit}

La cour siège quand un conflit se présente. En vue de la résolution du conflit et de la préparation du jugement, le jugement - qui est l'affirmation de l'univocité de la loi - doit être momentanément suspendu. Afin de distinguer le vrai du faux, on doit se servir de mesures adaptées à l'objet du conflit, de sorte que des principes juridiques universels puissent trouver une application. La cour ne s'ajuste donc pas à l'objet même du conflit, mais à la représentation de celui-ci. Aussi juste que puisse être cette représentation pour la plupart des cours, celles-ci se différencient toutefois quant à certains principes de mise en scène; la cour populaire des Grecs met l'individu face à l'individu et produit avec le jugement la différence asymétrique corps/esprit. Le théâtre romain des personnes se fonde sur l'asymétrie de la représentation des esprits, tandis que le droit moderne, dans ses développements, entend mettre tous les individus à portée du jugement; plutôt que d'individus, on pourrait ici parler de «biones» dont la sensualité est prise en compte par l'architecture de la procédure. Il se trouve en effet que, dans tous les cas, la capacité de se prononcer sur des parcours d'expérience précis est suggérée. Ces parcours sont toujours inscrits parmi les modèles architectoniques de la réalité qui constituent la structure fondamentale d'une topologie du droit. La possibilité de siéger, d'écouter différents cas, de présenter plusieurs versions de cas concrets, et d'exhiber la contingence des conflits au vu de leur expérimentabilité procédurale, est l'affaire de l'architecture judiciaire.

L'architecture judiciaire crée des oppositions et s'organise de telle sorte que cette différence des positions soit connue. Il est nécessaire que des éléments de la présentation qui virtualisent ce qui est présenté glissent au cœur de cette différence. Ils forment dans le conflit « l'universel concret » du cas, et par là même la décidabilité de ce cas. Chaque procédure doit en même temps composer avec cette équation propre de cas et préciser les limites de l'application du droit. Le tribunal expérimente l'antagonisme de la loi et du jugement.

Le jeu réciproque entre architecture et représentation permet au tribunal d'interpréter des scénarios comme sur une scène de répétition. En lien étroit avec ces scénarios apparaît maintenant un corps, dont la corporéité - à la différence de l'intention, c'est-à-dire à la différence de la fin d'une action dont on suppose le substrat - est fixée et, au sens propre, produite par le jugement. Seul le condamné a un corps. Au cours du procès apparaît en revanche « le corps inconnu », une chose dont on ne sait pas encore si elle peut, ou a pu, perpétrer certaines actions. La procédure judiciaire, 


\section{Labyrinthe, $n^{\circ} 23$}

contrairement à, et au-delà, de la légalité, établit par conséquent l'ignorance et l'improbable dans leur droit. L'évaluation (le jugement) met un terme à l'effet et constate le corps. La conscience du droit se construit chez ceux qui auparavant l'ignoraient et n'y prenaient pas part.

On doit, pour conclure, observer que dans le tribunal populaire des Grecs, à l'opposé de la cour d'assise de Hegel, ce n'est pas la participation des citoyens à l'application de leur droit, en tant qu'acte d'une liberté subjective, qui est centrale (il en aurait été ainsi, si des deux personnes qui s'opposent, les deux avaient également rendu le jugement). Ceux qui jugent représentent le public fortuit. Leur grand nombre répartit les conséquences d'une décision, ainsi que la séparation d'un état préalable de légalité, sur le plus grand nombre d'épaules possibles. Elle dégage les hommes, dans le plus grand nombre et la plus grande contingence possible, hors du cercle de ce qui, jusque-là, avait quelque valeur. L'autonomie n'est pas, n'en déplaise à Foucault, le produit de la subjectivation, de la soumission à une règle, mais le privilège du public qui bouleverse la loi, dans la mesure où il se place au-delà de la loi. Elle désincarne la multitude de ceux qui jugent.

\section{1 - Le droit comme événement}

Si jusqu'à l'ouverture de la procédure, la norme du droit est supposée obligatoire et compréhensible, avec l'ouverture de la procédure cette obligation est suspendue afin de légitimer la nécessité de la sentence du juge. La procédure se clôt avec l'institution du droit. Tout le temps que dure la procédure, ce qu'est le droit, ou plutôt ce qu'il va devenir n'est pas clair. D'abord, dans cet espace de liberté, il devient évident que le juge ne doit pas être juriste. Ensuite, le caractère de la décision que l'on devine arbitraire (ainsi que la possibilité qu'un jugement en contredise un autre) fait de la procédure juridique un exercice anarchique. C'est là que réside sa signification. Le droit est l'événement de ce renversement.

Traduit de l'allemand par Charles Ruelle et Guillaume Paugam*. Avec l'aimable autorisation de Wilhelm Fink Verlag.

\footnotetext{
* Nous remercions Élodie Cassan et Clément Orillard pour leur relecture attentive.
} 